\title{
Significados que construyen mujeres afrodescendientes frente al cáncer de mama y cuello uterino, atendidas en Medellín, Colombia
}

\author{
Meanings that build Afro-descendant women facing breast and cervical cancer, who were attended in Medellin, \\ Colombia
}

Sara Milena Ramos-Jaraba ${ }^{*}$ orcid.org/0000-0002-8638-5209

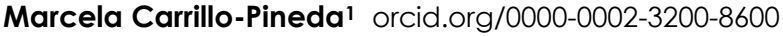

1 Facultad de Enfermería, Universidad de Antioquia. Medellín, Colombia.

Fecha de recepción: Octubre 31 - 2017

Fecha de revisión: Marzo 19 - 2018

Fecha de aceptación: Abril 13 - 2018

Ramos-Jaraba SM, Carrillo-Pineda M. Significados que construyen mujeres afrodescendientes frente al cáncer de mama y cuello uterino, atendidas en Medellín, Colombia. Univ. Salud. 2018;20(2):111-120. Dol: http://dx.doi.org/10.22267/rus.182002.115

\section{Resumen}

Introducción: La investigación sobre la salud de mujeres afrodescendientes es escasa en Colombia, especialmente cuando se trata de estudios que muestren relación entre etnia y cáncer. El cáncer de mama (CaMa) y cuello uterino $(\mathrm{CaCu})$ requieren ser investigados desde la voz de las mujeres afrodescendientes que lo padecen. Objetivo: Comprender los significados frente al $\mathrm{CaMa}$ o $\mathrm{CaCu}$ que construyen mujeres afrodescendientes atendidas en los servicios de salud de Medellín, Colombia. Materiales y métodos: Investigación cualitativa con enfoque etnográfico. Se realizaron entrevistas semiestructuradas a mujeres con $\mathrm{CaMa}$ y $\mathrm{CaCu}$ y grupos focales con población afrodescendiente sin cáncer; observaciones en citas médicas, quimioterapia y lugares cotidianos. El análisis fue guiado por el método de comparación constante. Resultados: Emergieron tres categorías: "El señor cáncer" que da cuenta de la enfermedad concebida como fatal y peligrosa, "La enfermedad desplaza", producto de la migración de las mujeres a Medellín para recibir tratamientos; y "Parte la vida en dos" por los cambios que conlleva la enfermedad. Conclusión: La enfermedad se concibió más allá de lo biológico al experimentar una alteración de la vida social, familiar y emocional. Se requiere mayor visibilidad del proceso salud-enfermedad de las afrodescendientes, por su rol ancestral como cuidadoras y forjadoras de unidad familiar.

Palabras clave: Cáncer de mama; cáncer de cuello uterino; mujeres afrodescendientes; significados; investigación cualitativa. (Fuente: DeCS, Bireme).

\begin{abstract}
Introduction: Research on the health of Afro-descendant women is scarce in Colombia, especially when it comes to studies that show the relationship between ethnicity and cancer. Breast cancer and cervix need to be investigated from the voice of Afro-descendant women who suffer from it. Objective: To understand the meanings facing breast or cervix cancer that are built by Afro-descendant women in the health services of Medellín, Colombia. Materials and methods: A qualitative research with an ethnographic approach was made. Semi-structured interviews were conducted for women with breast and cervix cancer and there were focal groups with Afro-descendant population without cancer with observations in medical appointments, chemotherapy and everyday places. The analysis was guided by the Constant Comparative method. Results: Three categories emerged: "Lord Cancer" which accounts for the disease conceived as fatal and dangerous, "the disease displaces", product of the migration of women to Medellin to receive treatments; and "it parts life in two" because of the changes that the disease brings. Conclusion: The disease was conceived beyond the biological part by experiencing an alteration of social, familiar and emotional life. There is a need for greater visibility of the health-disease process of the afro-descendants, or their ancestral role as caregivers and family unit builders.
\end{abstract}

*Autor de correspondencia

Sara Milena Ramos Jaraba

e-mail: sararamosj@gmail.com 
Keywords: Breast neoplasms; uterine cervical neoplasms; Afro-descendent women; meanings; qualitative research. (Source: DeCS, Bireme).

\section{Introducción}

El cáncer es una de las enfermedades que conlleva una carga psicológica muy fuerte y una poderosa dimensión metafórica, sobre todo porque ha estado socialmente construida como una enfermedad mortal; esta construcción ha hecho que el cáncer, se haya representado como una enfermedad tabú, en la que ha persistido su malignidad y fatalidad(1). Los tipos de cáncer que se originan en la zona reproductiva de la mujer, han tenido una connotación social mucho más negativa, ya que se relaciona con zonas del cuerpo que tienen que ver con la sexualidad y la imagen corporal, alterando el autoreconocimiento, las relaciones sociales, íntimas y emocionales(2).

En el mundo, el Cáncer de Mama (CaMa) y el Cáncer de Cuello Uterino $(\mathrm{CaCu})$ constituyen la primera y cuarta causa de muerte por cáncer en mujeres en regiones menos desarrolladas y en Colombia representan los cánceres de mayor mortalidad. Las tasas estandarizadas por edad estimadas en 2012, muestran que el CaMa tiene una tasa de incidencia de 35,6 casos por 100.000 mujeres-año y una tasa de mortalidad de 10 por 100.000 mujeres-año y el $\mathrm{CaCu}$ tiene una incidencia de 18,6 y una mortalidad de 8 por cada 100.000 mujeres respectivamente(3). Las tasas de mortalidad e incidencia a nivel mundial, también han mostrado diferencias de acuerdo a la etnia; por ejemplo estudios en Estados Unidos han encontrado que las tasas de incidencia por cáncer de cuello uterino son $45 \%$ más altas entre las mujeres afrodescendientes y $65 \%$ entre las hispanas, en comparación con las mujeres blanco/mestizas(4); con relación al cáncer de mama algunos estudios demuestran que las tasas son más altas en mujeres afrodescendientes jóvenes en comparación con mujeres de otras etnias $^{(5)}$. Por otro lado, la supervivencia, la oportunidad del diagnóstico y acceso a programas de prevención de cáncer entre mujeres afrodescendientes es baja, ya que se han identificado problemas de acceso a servicios de salud, la ausencia de seguro de salud y otros factores sociales y culturales que retrasan la búsqueda de atención( ${ }^{(6)}$.

Estudios realizados con mujeres afrodescendientes, revelan múltiples significados otorgados de manera individual y social al cáncer; así con relación al CaMa se ha encontrado que existen factores culturales relacionados con las creencias sobre la enfermedad, el miedo al cáncer y a la muerte, el desconocimiento frente a los efectos adversos de los tratamientos, las relaciones familiares, el soporte familiar y la espiritualidad, además múltiples barreras del sistema de salud para realizarse los tratamientos y seguimientos(7). En mujeres africanas con $\mathrm{CaCu}$, se encontró que el contagio de la enfermedad ha estado asociado al ejercicio de la sexualidad y con conductas sexuales socialmente inapropiadas para el género, como promiscuidad o prostitución, por lo que padecer $\mathrm{CaCu}$ conlleva una gran carga emocional adicional para ellas, asociada a la estigmatización sexual(8).

En el tema de cáncer y a diferencia de otros países, en Colombia son escasos los estudios que muestren disparidades entre grupos étnicos y desde el punto de vista cualitativo aún desconocemos las cosmovisiones, lo simbólico, subjetivo e intersubjetivo que se construye a partir del cáncer, situación ya investigada en países como África ${ }^{(9)}$. Esto requiere ser estudiado, entendiendo que los procesos de salud enfermedad son universales y operan en forma diferenciada en todas las sociedades y en los conjuntos estratificados que la integran, ya sea que se centre en un espacio comunitario o que englobe un grupo étnico(10).

Acercarse a este fenómeno contribuiría a que los profesionales de los programas y servicios de salud generales y especializados, brinden un trato diferencial y comprendan la influencia cultural de las mujeres en la forma de vivir su enfermedad. Por lo anterior el objetivo de este estudio consistió en comprender los significados construidos por mujeres afrodescendientes 
frente al cáncer de mama o cuello uterino, atendidas en Medellín, Colombia.

\section{Materiales y métodos}

Se realizó un estudio cualitativo con enfoque etnográfico(11,12), entre febrero de 2016 y abril de 2017, el cual permitió describir las creencias, prácticas, actitudes y formas de pensamiento de 14 mujeres afrodescendientes, entre los 33 y 77 años de edad, 9 de ellas diagnosticadas con CaMa y 5 con $\mathrm{CaCu}$, que fueron atendidas en instituciones prestadoras de salud en la ciudad de Medellín. Se excluyeron mujeres en fase terminal del cáncer y con un diagnóstico menor a un año por recomendaciones del comité de ética. Participaron adicionalmente 11 personas afrodescendientes sin cáncer, quienes compartieron sus opiniones respecto al fenómeno de estudio, lo cual fue muy útil, ya que este enfoque se apoya en la convicción de que la cultura donde están inmersos los sujetos crea y recrea las formas de pensamiento y las prácticas cotidianas de los mismos(12).

El muestreo por patrón común(13), se realizó a través de la estrategia de bola de nieve y mediante Instituciones Prestadoras de Salud o Empresas Promotoras de Salud del régimen subsidiado. Con estas participantes no se había tenido vínculos previos. El estudio contó con personal capacitado en investigación cualitativa.

Para obtener la información se hicieron 14 entrevistas semiestructuradas, de aproximadamente una hora cada una y grabadas en audio, con las mujeres que vivieron el fenómeno en sus lugares de residencia o en las casas de paso o albergue (lugar de estancia temporal en Medellín); a estas participantes se les aplicó un breve cuestionario sociodemográfico para su caracterización y para conocer los aspectos generales de sus condiciones sociales.

También se hicieron 35 observaciones participantes - 57 horas, en Medellín y Quibdó en los escenarios de la vida cotidiana de estas mujeres, entre ellos, sus lugares de residencia o estancia y durante el acompañamiento a algunas citas o procedimientos médicos. Las observaciones fueron plasmadas en el diario de campo y posteriormente transcritas en las fichas de observación.

Se realizaron dos grupos focales, el primero se organizó a través de una lideresa afrodescendiente, quién invitó a varias mujeres de su barrio, en el que asistieron 6 en total y el segundo se realizó en un hogar de paso, donde asistieron 5 participantes.

El análisis de los datos se realizó con la ayuda del Atlas ti versión 6.2; y fue guiado por el método de las comparaciones constantes a partir del cual emergieron los datos de forma inductiva, mediante un proceso de codificación que buscaba el entendimiento del fenómeno social y su profundización(14). El muestreo teórico permitió alcanzar la saturación, cuando los datos empezaron a repetirse y no había aportes nuevos(14). Durante la codificación abierta emergieron alrededor de 1455 unidades de significado y 210 códigos, los cuales se agruparon, según similitudes y diferencias, en tres categorías que apuntaban al objetivo, durante la codificación axial. La codificación selectiva contribuyó a integrar y refinar estas categorías, y a identificar la categoría interpretativa o trasversal de este estudio.

\section{Consideraciones éticas}

Este estudio fue aprobado por el comité de ética de la Facultad de Enfermería de la Universidad de Antioquia en acta № CEI-FE 2015-33, del 18 de marzo de 2016. Se siguieron los requerimientos de las Normas Científicas, Técnicas y Administrativas para la Investigación en Salud, según la Resolución 008430 del 4 de octubre de 1993 del Ministerio de Salud de Colombia; las normas internacionales que regulan la ética de la investigación (Declaración de Helsinki, 2008; Pautas de la CIOMS, 2002) y los requisitos éticos para la conducción responsable de la investigación clínica (Ezequiel Jonathan Emanuel). Las participantes firmaron un consentimiento informado. Se respetó su participación voluntaria, su confidencialidad utilizando seudónimos y se estableció una comunicación con ellas como interlocutoras 
válidas, reconociendo su experiencia con respecto al fenómeno de estudio. Ninguna de las participantes se negó a participar en la investigación.

\section{Resultados}

A partir del análisis de los datos, se encontró que los significados y las prácticas que las mujeres afrodescendientes participantes de este estudio, construyen sobre el CaMa y CaCu están dados por el contexto de la vivencia del cáncer. Si bien algunas mujeres habían escuchado sobre la enfermedad o tenían información previa, saben en realidad qué es el cáncer cuando lo viven en carne propia, es decir, cuando se acercan a la enfermedad desde la experiencia. Lo anterior también se evidencia en las diferencias entre los relatos de los participantes de los grupos focales y las mujeres.

Las mujeres construyen los significados del cáncer, en un espacio social aislado y un sistema de salud precario, como es Chocó o el Urabá Antioqueño; estos determinan en mayor medida el principal significado que las mujeres construyen frente al cáncer, en términos de fatal y peligroso, por lo que lo denominan "El señor cáncer". Para las mujeres que participaron en este estudio, el cáncer también es una enfermedad que "Parte la vida en dos", esta categoría da cuenta de los cambios en la vida de las mujeres antes y después del diagnóstico, pues la vida de las mujeres no vuelve a ser la misma. Otro de los significados que construyen las mujeres frente al cáncer, descrito en la categoría "La enfermedad desplaza", está relacionado con el hecho de tener que cambiar de residencia para obtener atención sanitaria, en una temporalidad que se vuelve casi permanente.

\section{El señor cáncer}

Esta categoría da cuenta de uno de los significados que las mujeres afrodescendientes construyen frente al CaMa o $\mathrm{CaCu}$. "El señor cáncer", es un calificativo que muestra el poderío y transformación que tiene la enfermedad en la vida de las mujeres participantes y de algún modo el miedo y respeto que lleva implícito la palabra cáncer.
Uno de los significados que han elaborado las mujeres participantes y el grupo afrodescendiente frente al cáncer, ha sido el de fatalidad o muerte. Hablar sobre el cáncer, o tan solo mencionarlo causa un pensamiento de muerte: "Cuando me hablan del cáncer de mama y cuello uterino me da escalofríos y pienso en la muerte de inmediato" (M-Grupo 01). Si bien el significado de fatalidad sobre el cáncer es común para todo el grupo, ser diagnosticada de la enfermedad es diferente para las mujeres, al ser la propia vida la que está en riesgo: "Dije me morí" (Gabriela).

Entre las razones por las cuales consideraban el cáncer como una enfermedad fatal, las participantes mencionaron que: "Como es una enfermedad que siempre yo había escuchado que la gente que padecía eso se moría, yo dije: hasta aquí, ya fueron mis últimos días" (Victoria). Por otro lado, las participantes mencionan que en Chocó y Urabá la enfermedad se diagnosticaba en etapas tardías, debido a la falta de acceso a los servicios de salud y a que el conocimiento y los medios para diagnosticar la enfermedad eran limitados: "Porque el cáncer para mí era muerte, porque yo he visto mucha gente morir de cáncer. Porque en el Chocó el cáncer era como que la gente se lo detectaban bien tarde y cuando se lo detectaban ya estaba era muriéndose" (Gabriela).

También se evidenció que el significado de fatalidad ha tenido transformaciones y la cura de la enfermedad no se concibe tan lejana, al haber posibilidad de atención en Medellín, sin embargo existe una concepción del cáncer como terrible y peligrosa, por las consecuencias que deja para quienes lo padecen. Este significado de terrible y peligroso, se presenta, pues es una enfermedad que "al principio no tiene síntomas" (P01-Grupo 01), "invade el cuerpo en silencio" (P07-Grupo 02), porque "es una enfermedad que destruye" (P02-Grupo 02) y por características que se identificaron tanto al pasar la experiencia del cáncer, como por las creencias, imaginarios y comentarios, construidos social y culturalmente.

Entre las principales características que se le da a la enfermedad es la capacidad de ser silenciosa, por lo cual pasa desapercibida ante 
quien la padece y los que la rodean, además de consumir el cuerpo lentamente. Este significado no fue solo dado por las mujeres con el diagnóstico de cáncer, sino que también se encontró en los grupos focales, como muestra el siguiente fragmento: "Es una enfermedad silenciosa, que va consumiendo el cuerpo" (Mujer, Grupo 01).

El cáncer es concebido para las mujeres como una enfermedad peligrosa, pues según lo que se puede notar en sus relatos les destruye la vida, pues según lo comenta uno de los participantes de un grupo focal es una enfermedad que siempre termina mal: "Sí, es una enfermedad cara, mortal, mejor dicho es una enfermedad que prácticamente sino tiene cura deja a los familiares en ruina, se muere el paciente y vive mal uno" (Hombre, Grupo 02).

\section{Parte la vida en dos}

La experiencia de la enfermedad es un evento que parte sus vidas en dos, no solo por lo que ocasiona en términos biológicos y físicos, sino porque ocurre un cambio en sus vidas a nivel laboral/económico, sentimental, y familiar, por lo que nada vuelve a ser igual.

Las mujeres narran que al ser diagnosticadas de cáncer, no vuelven a ser las mismas de antes, teniendo en cuenta que por causa de la enfermedad no pueden trabajar, ya sea porque están lejos y porque físicamente la enfermedad o los tratamientos no lo permiten. Esta realidad empeora su situación económica y crea una dependencia de sus familiares o hijos, quienes asumen estas responsabilidades: "Pues yo hoy en día ya no soy la que era, porque ya no puedo estar trabajando, a veces que me aburro, me azaro [desespero] porque tengo que esperar que todo me lo den. Que si mis hijos no me dan pa' yo comer no, entonces eso hoy en día me tiene triste" (Ramona).

La relación con los hijos es otro aspecto de sus vidas que se altera por la enfermedad, ya que por los múltiples viajes y el tratamiento tienen que dejarlos a cargo de familiares $\mathrm{u}$ otros hijos mayores, teniendo un sentimiento de abandono hacia ellos, como se menciona en el siguiente testimonio: "Como dice el dicho ya esto es una experiencia vivida, que uno ya la adquirió para contarle a otros, porque esto es una cosa que, quien ya tiene esta enfermedad, tiene una vida, que si uno tiene los hijos los dejó botados a lo que Dios quiera"(Ramona).

No ser la que era antes, también implica algunos aprendizajes adquiridos por las mujeres en su transitar con la enfermedad. Si bien, en general, esta experiencia se concibe como terrible o negativa, muchas mujeres también la asumen como potencialidad u oportunidad de cambio, tanto para su transformación personal, como en la relación con la familia: "Yo he cambiado mucho, lo que yo era antes, mi marido me dice que, ;hay vos como has cambiado, ahora miras a las personas diferente, vivís pendiente de los vecinos!, ¡ay mijo!, la vida lo pone a uno a cambiar así, uno tiene que cambiar porque con las enfermedades tiene para cambiar" (Camila).

Los problemas en la relación de pareja, también se conciben como un cambio a partir de la enfermedad, las mujeres mencionan que los largos periodos de tiempo fuera de su hogar, provocaron consecuencias en su vida de pareja. En su mayoría las mujeres sufrieron separaciones, y se mencionan las infidelidades, no poder tener relaciones sexuales y los cambios físicos, derivados de la mastectomía, como otras causas: "Entonces, como no podía hacer coito con el marido, yo creo que eso fue lo que lo alentó para el irse a buscar otra mujer, aunque él me dice que no, pero sí tiene su otra mujer, él todavía la tiene" (Camila).

Los cambios físicos en el cuerpo son evidentes a lo largo del tratamiento del cáncer y después, en especial en el caso de mujeres que fueron mastectomizadas o aquellas que recibieron quimioterapia y sufrieron la caída del cabello. Lo anterior deja una serie de secuelas físicas y psicológicas, con las que se tiene que convivir y buscar estrategias para afrontarlas: "Ay vea mejor dicho con gorro, yo no salgo a la calle sin el gorro" (Sandra). Para algunas mujeres no tener un seno era una situación que si bien era dolorosa, se podía manejar, a través del uso de: esponjillas, prótesis removibles, u otros objetos. Por el contrario, no tener el cabello era difícil de 
disimular, hecho que se concibió como lo más duro:..."Pero el seno, tu puedes disimularlo con algo, no sé, poniéndole un relleno al seno, pero el cabello es duro" (Lucía).

"Yo no siento tristeza por el seno, más dolor siento por mi pelo" (Sandra).

Entre estos cambios, es de resaltar que las mujeres mastectomizadas no estuvieron muy interesadas en realizar la reconstrucción del seno, ya sea por temor o por malas experiencias propias o de otras mujeres conocidas: "Sabe por qué me he vuelto para atrás con eso [reconstrucción mamaria]?, porque he visto amigas mías que se han mandado a construir el seno y eso se les ha alborotado, eso ahí se les ha alborotado otra vez" (Ramona).

\section{La enfermedad desplaza}

Esta categoría es el resultado del sistema de salud precario, ya que las mujeres, en particular las que viven en Urabá y en el Chocó, debieron irse de sus lugares de origen cuando fueron diagnosticadas de cáncer, dejando sus hogares, sus familias y su trabajo, para desplazarse a Medellín a buscar la cura de su enfermedad. "Pues la enfermedad me desplazó buscando la cura, para ver si Dios me salvaba, porque él me destinó que para acá tenía que venir yo a buscar la cura y acá me trajo él y acá he seguido viniendo y él sigue trayéndome [a Medellín] y llevándome otra vez [a Quibdó]" (Ramona).

Este desplazamiento ocurre generalmente cuando los profesionales de la salud en Chocó y Urabá reconocen que es necesaria la remisión a otros niveles de complejidad en Medellín. Dicho desplazamiento en ocasiones ocurre de manera súbita, ya que las mujeres deben venir a la ciudad sin dinero, sin tener un lugar donde quedarse y en ocasiones con la poca ropa que tenían en el momento de la remisión, como revela el siguiente testimonio: "Un especialista me dijo: ¿a usted le han dicho que tenía? no doctor no me han dicho que tengo iah bueno!, usted va ir para Medellín ¿oyó? ¿Para Medellín? ¡Pero yo no tengo plata! [Médico]: No, arréglese que se va, no me importa si usted tiene o no tiene, nadie le ha preguntado por nada" (Sandra).
Llegar a Medellín a realizarse el tratamiento, genera incertidumbre y angustia, al enfrentarse a una ciudad desconocida. Como en el caso de Sandra, también hay otras mujeres que son remitidas por la EPS, sin que se resuelva la situación de alojamiento: "Me dieron los pasajes hasta el aeropuerto y ahí quedé. Donde mis hijos no me hubieran dado plata $\mathrm{pa}^{\prime}$ moverme del aeropuerto a buscar donde alojarme, no sé qué hubiera hecho yo ahí. Apenas hasta ahí [aeropuerto] muy mal hecho, eso para mí es mal hecho, una persona que no conoce una ciudad como Medellín... váyase, móntese en un avión y ya llegue al aeropuerto y ¿para dónde va a coger?" (Mary).

El motivo de este desplazamiento es poder buscar la cura de la enfermedad, al aceptar que este traslado trae beneficios relacionados con la prestación del servicio, la recuperación y en general la solución a los problemas de salud. Sentirse desplazadas, tiene otras consecuencias e implica también dejar a la familia. Su permanencia en Medellín por tiempo prolongado, hace que dejen a sus hijos, convirtiendo esta experiencia en una de las más difíciles: "En el proceso de tratamiento lo más duro fue estar alejada de mi familia, la verdad es que nunca en mi vida me había alejado de mi hijo menor, así tanto tiempo no, casi siempre he estado con él, eso sí me pareció un poquito pues muy duro" (Astrid).

En el desplazamiento causado por la enfermedad, las mujeres deben buscar un lugar donde vivir en Medellín, mientras se realizan los tratamientos médicos y controles. Algunas de las mujeres, los primeros días o meses llegan a las casas de familiares y amigos que se encuentran en la ciudad, como una opción temporal a su problema, sin embargo otras mujeres por el contrario no se pueden quedar en un solo sitio y deben andar de casa en casa: "Yo estuve hospitalizada, estuve ahí 4 días, de ahí a una casa de un tío de mis hijas, de ahí me fui con una nuera que vivía por allá lejos, por allá paré como 3 semanas. Ella se iba para Chocó y me dejó en esa casa, de ahí paré como 8 o 15 días más" (Ramona). 
Aunque las mujeres cuentan con el apoyo de sus familiares o amigos en una primera etapa de la enfermedad, la situación se complica cuando el tratamiento se está haciendo largo e inician los problemas de convivencia, las dificultades económicas y la falta de apoyo, por lo que ven la necesidad de solicitar un hogar de paso, con el fin de no molestar más a sus parientes, como se menciona en el siguiente fragmento: "Usted sabe que los paisanos, eso desde que usted llegó lo primero que preguntan es cuando se va y si usted no llega con plata no" (Mary).

Como se mencionó en la categoría "parte la vida en dos", los problemas económicos se derivan a partir del diagnóstico, sin embargo el desplazamiento hacia Medellín, implica mayores gastos de bolsillo, al tenerse que costear: los utensilios de aseo, los pasajes, algunos alimentos y dejar de trabajar para realizarse el tratamiento, por lo tanto estar en otra ciudad se vuelve costoso: "El estrés me iba a matar, todos los días tenía que coger las 2 busetas porque la EPS esos pasajes si no los da, todos los día me tocaba coger las 2 busetas, bajarme de allá de Llanadas hasta aquí al centro de aquí coger la del poblado, todos los días me tocaba sagradamente amanecer con esos ocho mil pesos" (Mary).

En las observaciones realizadas en las casas de paso, y la asistencia a talleres de mujeres con cáncer en este mismo hogar, se observó que existe un deseo permanente de regresar a sus hogares y "comenzar una vida normal" [NC, 15 de junio de 2016; hogar de paso, Medellín]. En las conversaciones con las mujeres, todas refieren un sentimiento de vacío, abandono, y una contradicción al querer volver, pero al mismo tiempo sentir temor de que en Chocó y Urabá regrese la enfermedad y no haya forma de atenderla, ante los problemas de los servicios de salud: "Mi hermano me dice que me quede viviendo acá en Medellín, que aguante, porque sino me muero en Chocó" (Carmen).

\section{Discusión}

Tal como se describió en el apartado anterior los significados que las mujeres afrodescendientes construyen alrededor del CaMa y $\mathrm{CaCu}$ ocurren en un espacio social adverso, lo que influye en que la enfermedad sea nombrada como el "señor cáncer", dada su fatalidad y peligrosidad. La experiencia de tener cáncer le "parte la vida en dos" a la mujeres, sobre todo a aquellas quienes son "desplazadas por la enfermedad". Lo anterior evidencia que la construcción de significados está influenciada por un sistema de salud que es excluyente con la población étnica y que obedece a un modelo de desarrollo centro-periferia, donde los servicios de salud se concentran en las grandes ciudades atendiendo a la lógica del sistema de mercado y a la ley de oferta y demanda, situación que afecta de manera desproporcionada a los habitantes de departamentos relegados del "desarrollo" nacional(15,16).

Según un informe de la Organización Panamericana de la salud(17), la atención en salud en Chocó presenta múltiples barreras de acceso, debido a la dispersión geográfica, inadecuadas condiciones de uso de los servicios de salud, el mal estado de las vías terciarias, la violencia, las precarias condiciones de infraestructura física y tecnológica que afectan el modelo de atención. En esta investigación se evidenció, poca oferta de servicios de salud en Chocó, ya que las mujeres solo accedían a centros de salud y en general primeros niveles de atención, siendo imposible diagnosticar o atender enfermedades como el cáncer. Lo anterior también se relaciona con que la mayoría de las mujeres pertenecían al régimen subsidiado y que la red de prestación para éste tipo de población es limitada.

Con relación a la migración en salud, un estudio realizado en varias ciudades del país, encontró que el principal obstáculo para la atención de mujeres con CaMa, fue la necesidad de desplazarse a otra ciudad para recibir tratamientos y además pagar por los medicamentos(18). Si bien Colombia cuenta con un plan para el control del cáncer, se ha encontrado que la atención de esta enfermedad es fragmentada, dispersa y se concentra en las grandes ciudades, siendo insuficiente ante la complejidad geográfica - poblacional. Por lo anterior las regiones más pobres carecen de una red de atención oncológica(19). 
Para las mujeres de este estudio, los significados que construyen sobre la vivencia del cáncer, se traduce en una serie rupturas, que causaron un quiebre en sus vidas y se relaciona con los cambios que se dan a partir del diagnóstico del cáncer. Se podría decir que el principal quiebre ocurre en las mujeres que deben migrar y abandonar todo lo que son en términos materiales y espirituales, esto significa dejar la casa, los hijos, la familia, los vecinos, el trabajo, sus prácticas, su alimentación, y su independencia, para viajar a Medellín a curarse.

Lo anterior es una situación estrechamente ligada a la inequidad geográfica y al sistema de salud precario, que limita el acceso y atención en salud a quienes necesitan un tratamiento oncológico, razón por la cual para las mujeres de este estudio llegar a Medellín, se concibe como la única solución a su problema de salud. Posiblemente si en Chocó y Urabá contaran con servicios de salud cualificados y adecuados para atender este tipo de enfermedad, las mujeres no tendrían que romper con su vida. Estar lejos de casa por culpa de la enfermedad, significa para muchas mujeres tener que dejar a sus hijos, hecho entendible por el rol que tiene la mujer como cuidadora en la sociedad. Los hallazgos de un estudio similar realizado con mujeres provenientes de diferentes regiones de Colombia y del régimen subsidiado, que se realizaban tratamientos para el cáncer y eran acogidas en un albergue en Bogotá, también mostraron que las relaciones familiares y en especial estar lejos de los hijos fueron expresados como un evento que afecta sus vidas, por lo cual desearían poder compartir con ellos en esta dura etapa de la enfermedad(20).

Las relaciones de pareja se convierten en otra ruptura a partir del diagnóstico del cáncer, situación ya documentada en otros estudios alrededor del mundo. Estos revelan problemas de tipo sentimental, y sexual, a partir del tratamiento, en especial en mujeres mastectomizadas, o en el caso del cáncer de cuello uterino por la estigmatización que tiene la enfermedad al estar asociada al contagio del Virus del Papiloma Humano(21,22). En este estudio, los problemas con la pareja se acentúan por la dinámica de las relaciones familiares y la migración de las mujeres. Desde el momento en que enferman y faltan en sus hogares, inician las rupturas con los hombres, pues dada su figura de autoridad, socialmente establecida, ellos no están dispuestos asumir tareas domésticas y de cuidado, pues desde su perspectiva, estas le han sido atribuidas a la mujer, con base en la división sexual de trabajo(23).

Los cambios con relación al cuerpo, han sido documentados en otras investigaciones, el malestar físico causado por los tratamientos, también ha sido reportado por otros estudios cualitativos, siendo la caída del cabello y la mastectomía sus principales consecuencias(24). Desde el punto de vista étnico no se encontraron muchas diferencias al comparar este estudio, con otros realizados en mujeres no afrodescendientes. Sin embargo es de resaltar que las diferencias se encuentran en la importancia que tiene el cabello para las mujeres afrodescendientes, en comparación con la pérdida de otros órganos como el seno o la matriz. La experiencia de la caída del cabello se concibe como un hecho traumático, vergonzoso o indeseado, además de no existir mayor interés en realizar la reconstrucción mamaria.

En investigaciones conducidas con mujeres Colombianas del área rural diagnosticadas de cáncer, se encontraron algunos hallazgos similares relacionados con los malestares físicos. Para estas mujeres la reconstrucción del seno no era prioridad, mientras que la caída del cabello las afectaba de manera significativa(25). Otros estudios realizados con mujeres africanas también sugieren múltiples preocupaciones sobre la imagen corporal, y salud sexual( ${ }^{(9)}$. Se pudiera entender entonces que para las mujeres afrodescendientes la caída del cabello es un hecho traumático, al ser éste la representación de su identidad y un símbolo cultural, evidenciado en los peinados o la extensión del cabello(26). Lo anterior demuestra que los significados de la auto-imagen son variados e influidos por los contextos culturales.

Para terminar, se sugiere incentivar un trabajo intersectorial en la atención del cáncer de 
mujeres afrodescendientes, que garantice el acceso a los servicios de salud, vivienda, alimentación, transporte y en general condiciones de vida dignas, en especial para aquellas que vienen de otros municipios o departamentos a realizarse el tratamiento para el cáncer en Medellín. También se requiere desde el personal de salud una atención diferencial, con pertinencia cultural, teniendo en cuenta los significados, las prácticas y las creencias que tienen las mujeres afrodescendientes frente al cáncer, las cuáles pueden considerarse de suma importancia en los procesos de educación y atención de la enfermedad.

\section{Conclusiones}

Las mujeres participantes de este estudio han construido el significado del cáncer más allá de lo biológico, al experimentar una alteración de la vida social, familiar y emocional, a raíz de la enfermedad, además de identificar su relación con el espacio social y geográfico en el que viven, que de una u otra manera las determina.

Se hace evidente la presencia de un modelo de atención en salud inequitativo, que empeora en espacios sociales con población de minorías étnicas, creando unas opresiones en las mujeres y agudizando su situación económica y social. Entre los resultados más relevantes del estudio se encuentran las inequidades en salud y de espacio geográfico en el departamento del Chocó y Urabá, las cuales generan un desplazamiento de las mujeres a Medellín, en busca de la cura de la enfermedad.

Es importante generar mayor visibilidad del proceso salud-enfermedad de las mujeres afrodescendientes, ya que ellas juegan un papel fundamental en la cosmovisión de la cultura afrodescendiente, pues son quiénes tienen un rol ancestral como cuidadoras y forjadoras de unidad familiar para la preservación de prácticas de cuidado de la salud, de ellas mismas y de la comunidad.

\section{Agradecimientos}

Agradecemos al grupo de investigación de promoción de la salud de la Facultad de
Enfermería de la Universidad de Antioquia y a la Primera Convocatoria de Becas de Maestría de la Universidad de Antioquia.

\section{Conflicto de interés}

Las autoras declaran que no existen conflictos de interés.

\section{Referencias}

1. Cortés C. Cáncer de cuello uterino: una enfermedad de mujeres con perspectiva de género. Reflexiones para el trabajo en salud pública. Florianópolis: Fazendo Gênero 10 - Desafios Atuais dos feminismos; 2013. p. 10.

2. Ferreira SM de A, Gozzo T de O, Panobianco MS, SantosMA dos, Almeida AM de. Barriers for the inclusion of sexuality in nursing care for women with gynecological and breast cancer: perspective of professionals. Rev Lat Am Enfermagem. 2015;23(1):82-9.

3. Ferlay J, Soerjomataram I, Ervik M, Dikshit R, Eser S, Mathers C, et al. GLOBOCAN 2012 v1.0, Cancer Incidence and Mortality Worldwide: IARC Cancer Base No. 11 [Internet]. Lyon, France: IARC; 2013. Available from: http://globocan.iarc.fr, accessed on 31/10/2017.

4. Rauh-Hain JA, Clemmer JT, Bradford LS, Clark RM, Growdon WB, Goodman A, et al. Racial disparities in cervical cancer survival over time: Racial Disparities in Cervical Cancer. Cancer. 2013;119(20):3644-52.

5. Shoemaker ML, White MC, Wu M, Weir HK, Romieu I. Differences in breast cancer incidence among young women aged 20-49 years by stage and tumor characteristics, age, race, and ethnicity, 2004-2013. Breast Cancer Res Treat [Internet]. 2018 [citado 19 de marzo de 2018]; Disponible en: http://link.springer.com/10.1007/s10549-018-4699-9

6. Iqbal J, Ginsburg O, Rochon PA, Sun P, Narod SA. Differences in Breast Cancer Stage at Diagnosis and Cancer-Specific Survival by Race and Ethnicity in the United States. JAMA. 2015;313(2):165.

7. Torres E, Dixon C, Richman AR. Understanding the Breast Cancer Experience of Survivors: a Qualitative Study of African American Women in Rural Eastern North Carolina. J Cancer Educ. 2016;31(1):198-206.

8. Birhanu Z, Abdissa A, Belachew T, Deribew A, Segni H, Tsu V, et al. Health seeking behavior for cervical cancer in Ethiopia: a qualitative study. Int J Equity Health. 2012;29:11-83.

9. Maree JE, Kaila I. Zambian Women's Experiences and Understanding of Cervical Cancer: A Qualitative Study. Int J Gynecol Cancer. 2014;24(6):1065-71.

10. Menéndez E. La enfermedad y la curación?` Qué es medicina tradicional. Alteridades. 1994;4(7):71-83.

11. Denzin N, Lincoln Y. El campo de la investigación cualitativa. Vol. 1. Barcelona: Gedisa Editorial; 2012. $365 \mathrm{p}$.

12. Gúber R. La etnografía: método, campo y reflexividad. Bogotá: Grupo Editorial Norma; 2001.

13. Patton M. Qualitative research and evaluation methods. 3a ed. United States: Sage Publication; 2002. 554 p. 
14. Strauss A, Corbin J. Bases de la investigación cualitativa. Técnicas y procedimientos para desarrollar la teoría fundamentada. Medellín: Universidad de Antioquia; 2002. $340 \mathrm{p}$.

15. Acuña C, Marina N, Mendoza A, Emmerick ICM, Luiza VL, Azeredo TB. Determinantes sociales de la exclusión a los servicios de salud ya medicamentos en tres países de América Central. Rev Panam Salud Pública. 2014;35(2):128-135.

16. Rodríguez A. S. Monopsony power in the market for health insurance in Colombia. Rev Econ Caribe. 2015;(15):121-52.

17. Organización Panamericana de la Salud. Salud efectiva para pueblos dispersos: Modelo de Atención en Salud. Departamento del Chocó [Internet]. Colombia: OPS; 2010. p. 36. Disponible en: http://www.paho.org/col/index.php?option=com_cont ent\&view=article\&id=929:modelo-de-atencion-ensalud-departamento-del-choco\&Itemid=361

18. Charry VD, Constanza L, Carrasquilla G, Roca-Garavito S. Equidad en el acceso al tratamiento para el cáncer de mama en Colombia. Salud Pública México. 2009;51:246-253.

19. Vergara-Dagobeth E, Suárez-Causado A, Gómez-Arias RD. Plan Control del cáncer en Colombia 2012-2021. Rev Gerenc Políticas Salud. 2017;16(33):6-18.

20. Lafaurie MM, Castiblanco BDR, González Díaz JM, Jiménez Tamayo DM, Moreno LB, Ramírez L del P, et al. Mujeres en tratamiento de cáncer, acogidas por un Albergue de Apoyo: circunstancias y perspectivas de cuidado de Enfermería. Rev Colomb Enferm. 2009;4(4):61-72.

21. Vermeer WM, Bakker RM, Kenter GG, Stiggelbout AM, ter Kuile MM. Cervical cancer survivors' and partners' experiences with sexual dysfunction and psychosexual support. Support Care Cancer. 2016;24(4):1679-87.

22. Fouladi N, Pourfarzi F, Dolattorkpour N, Alimohammadi S, Mehrara E. Sexual life after mastectomy in breast cancer survivors: A qualitative study. Psychooncology. 2018;27(2):434-41.

23. González NM. Maternidades y paternidades afrocolombianas en Cali $\mathrm{y}$ El Valle. Hist Espac. 2012;(38):42-62.

24. Jaman-Mewes P, Rivera MS. Vivir con cáncer: una experiencia de cambios profundos provocados por la quimioterapia. Aquichan. 2014;14(1):20-31.

25. Lafaurie MM. Mujeres con cáncer de seno: experiencias y significados. Rev Colomb Enferm. 2016;7(7):12-21.

26. Hellebrandová K. Escapando a los estereotipos (sexuales) racializados: el caso de las personas afrodescendientes de clase media en Bogotá. Rev Estud Soc. 2014;(49):87-100. 